\title{
Morphology and morphometry of bicuspid valve in human fetal cadavers
}

\section{Abstract}

A preliminary study of certain morphometric parameters of Bicuspid valve was undertaken in 40 aborted fetuses of 16 weeks to full term to provide a database that will be of help to cardiac surgeons to rule out abnormal measurements. AV circumference, frontal and sagittal dimensions, width of anterior cusp and AV circumference increased significantly in males. Width of posterior cusp increased significantly in both sexes. A correlation coefficient of all fetal bicuspid parameters positively correlated with each other. Bicuspid valve dimensions provide new information about its normal and abnormal function that will be of use in valve replacement surgeries.
Volume 3 Issue 4 - 2017

\section{Kishore Naick D, Subhadra Devi Velichety, Thyagaraju K}

Sri Padmavathi Medical College for women, India

Correspondence: Subhadra Devi Velichety, Professor of Anatomy, SVIMS, Sri Padmavathi Medical College for women, Tirupati,Andhra Pradesh, India, Email sdvelichety@hotmail.com

Received: January 12, 2017| Published: April 24, 2017

\section{Introduction}

Anatomy of bicuspid valve is complex is altered by various disease states. Data on dimensions of bicuspid valve may be of interest to anatomists and cardio-thoracic surgeons to assess the exact mechanical reason for valve insufficiency. ${ }^{1}$ Anatomists and researchers are frequently interested to study bicuspid valve because of its clinical importance in mitral valve repairs and in severe cardiac malformations. There are many diseases of the heart which affects the valves and result in valve insufficiency. They are stenosis and regurgitation of the valves or prolapse of the leaflets. Mitral valve diseases require repair of the valve and when repair is not possible, valve replacement surgery will be attempted. ${ }^{2}$ In the literature anatomical studies on mitral valve morphology and morphometry in adults based on observations in isolated clinical cases were reported. Due to its clinical significance, paucity of anatomic studies and no reports on the morphology and morphometry of bicuspid valve parameters in fetal hearts the present study was conducted.

\section{Materials and methods}

This study was carried out on 40 spontaneously aborted human fetal cadavers of 16weeks to full term gestational age and both the sexes for observations on morphological and morphometric parameters of bicuspid valve in heart specimen using paquimeter (Figure 1). The fetuses are assigned into Group-I (16-29weeks) and Group-II (30weeks into Full term) according to their gestational age.

The thoracic cage was opened following the procedure described in the literature (Figure 2). ${ }^{3}$ Middle mediastinal was exposed after removal of the lungs. The pericardium covering the heart was slit open from the diaphragm to the aorta and the heart was exposed after reflecting pericardium. By lifting the apex of the heart the pulmonary veins were excised at the place where they form the boundary for oblique pericardial sinus. Finally the serous pericardium was reflected to separate the heart from its position. Then a deep cut $2 \mathrm{~cm}$ to the left of anterior interventricular groove parallel to the left of interventricular septum was made. After opening the left ventricle to the ascending aorta, the bicuspid valve was exposed.

Atrioventricular circumference was measured (Figure 3) using a paquimeter. The anterior and posterior cusps of mitral valve were

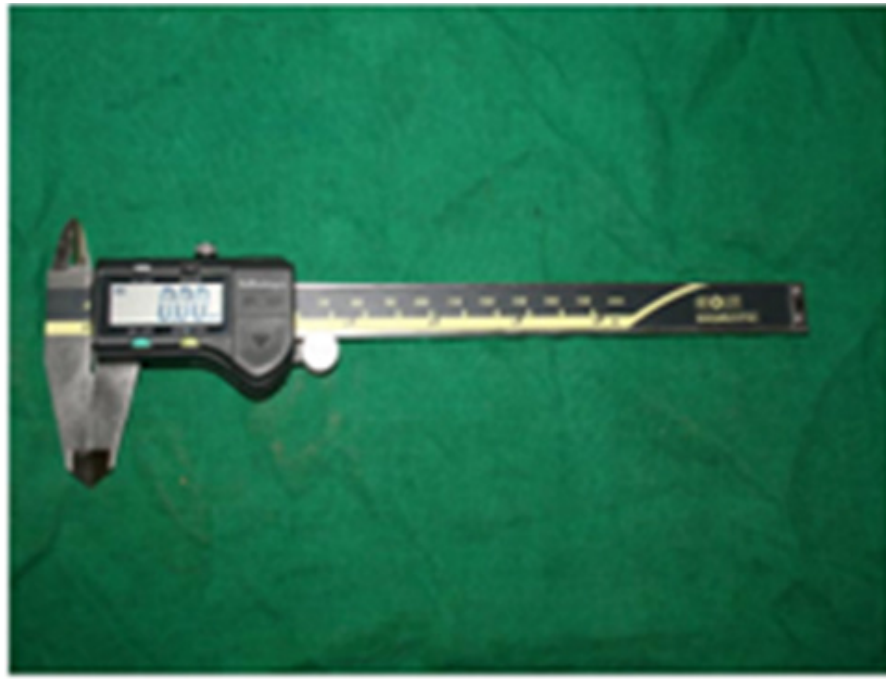

Figure I Paquimeter.

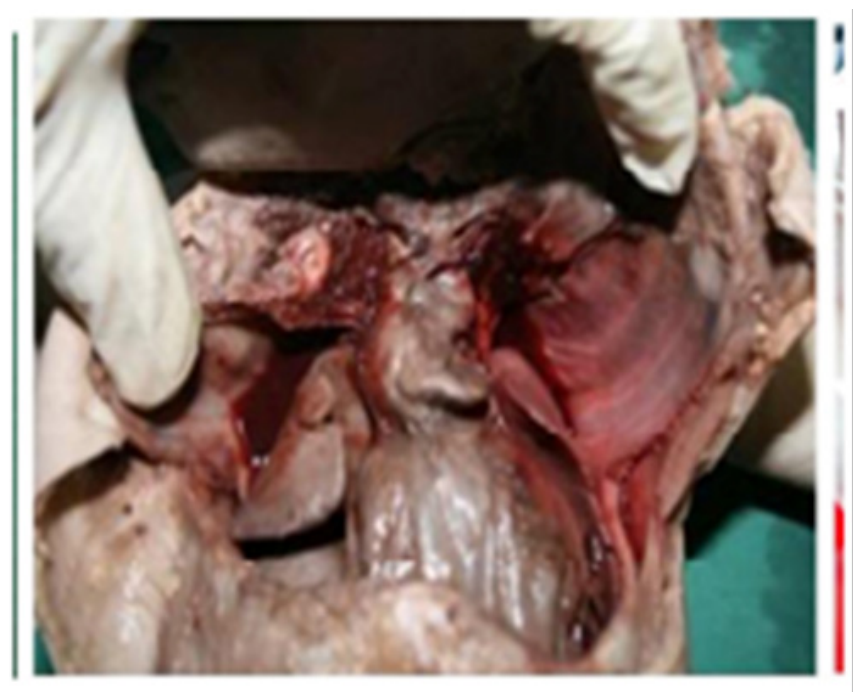

Figure 2 Opening of Thoracic cavity. 
identified (Figure 4). The bicuspid valve along with annulus, chordae tendinae and papillary muscles were removed by dividing its ring at the lateral commissure and by half splitting the medial papillary muscle mass as described in literature. ${ }^{3}$

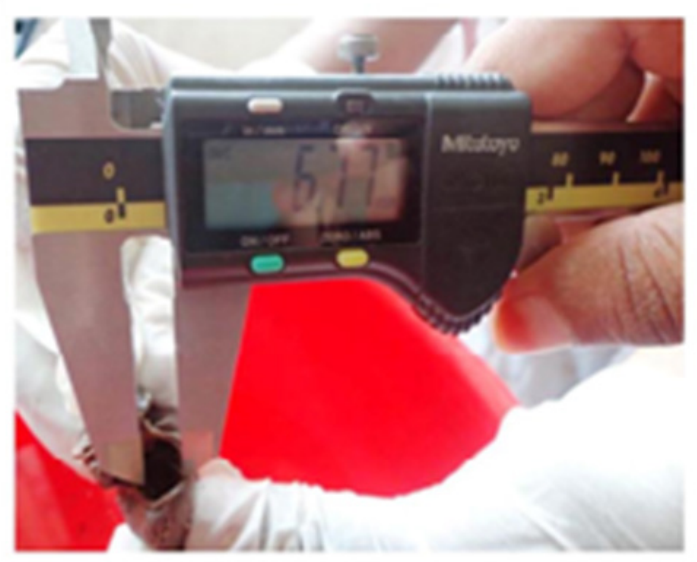

Figure 3 Showing measurement of atrioventricular orifice circumference.

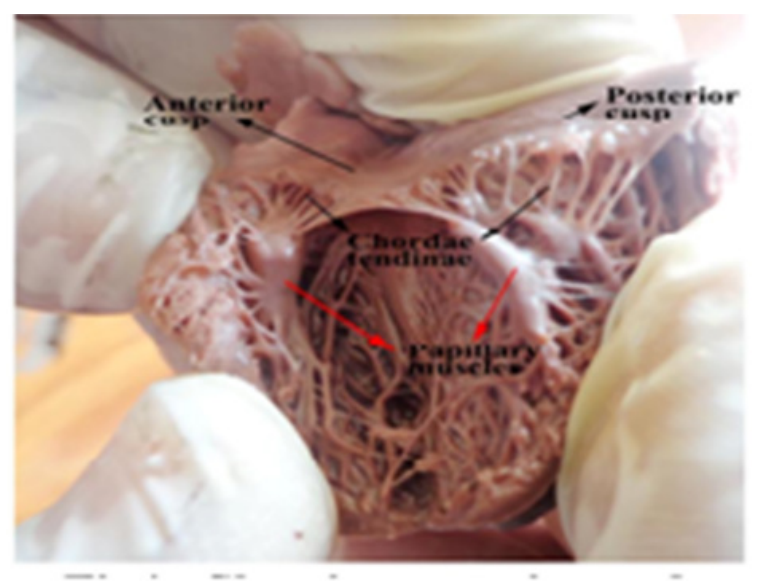

Figure 4 Showing anterior and posterior cusps, papillary muscles and chordae tendinea.

The following morphological parameters of Bicuspid valve were observed:

i. Cusps

ii. Chordae tendinae

iii. Papillary muscles

The following morphometric parameters of the bicuspid valve of fetal heart were defined and measured using paquimeter following the method of Skwarek et al. ${ }^{4}$

i. Basal Width of the cusps: greatest distance between the commissures (Figure 5).

ii. Depth of the cusps: greatest distance between the fibrous ring and the free edge of the cusps (Figure 6).

For the localization of the cusps, the atrioventricular fibrous ring was divided into two regions.

a. Anterior region: the distance between the commissures and the projection of the left margin in the fibrous ring.

b. Posterior region: distance of the last points from the inter ventricular septum and also the distance are measured from cusp to the chordate tendon attachment up to papillary muscle in bicuspid valve.

A. Attachment length of anterior and posterior leaflets.

B. Frontal dimension: measured from the commissure between the leaflets of the bicuspid valve along the axis of the left atrioventricular orifice to the sharp margin of the left ventricle.

C. Sagittal dimension: measured perpendicular to the frontal dimension at the midpoint of its length. ${ }^{4}$

D. Atrial circumference of the bicuspid valve attachment.

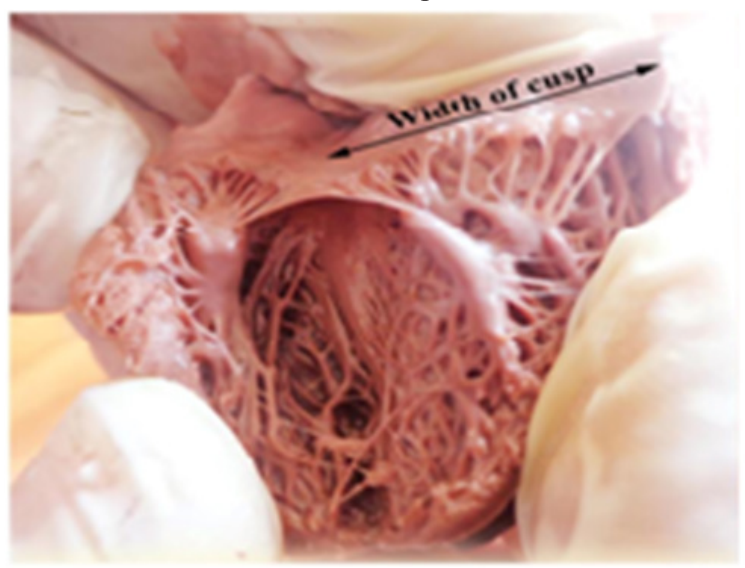

Figure 5 Measurement of width of cusp-Bicuspid valve.

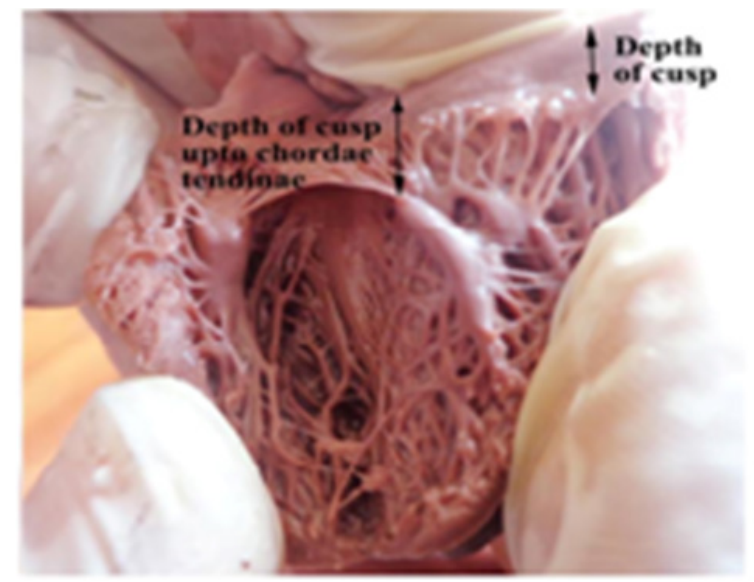

Figure 6 Measurement of depth of cusp.

On the basis of the above measurements a triangle and an ellipse were constructed to determine the shape of atrioventricular orifice. The triangle was marked using dimensions 2-4 and an ellipse using dimensions 5 and 6 mentioned above to determine the shape of the bicuspid valve. The areas of these geometric figures were calculated according to the following formulae,

For the triangle: $1 / 2 \times$ base $\times$ height and for the ellipse: $3.14 \times a \times b$

\section{Statistical analysis}

The data were analyzed with MED Calc R version 12.2. All results are shown as a Mean \pm Standard Deviation. Width and depth of the corresponding cusps were compared by unpaired t test. Pearson's correlation coefficient test was used to analyze the relation between the Mean distance and the annular diameter. 


\section{Results}

In the present study a total of 40 fetal hearts of both the sexes was categorized into two gestational age groups for observing morphology and morphometry of bicuspid valve as shown in Graph 1.

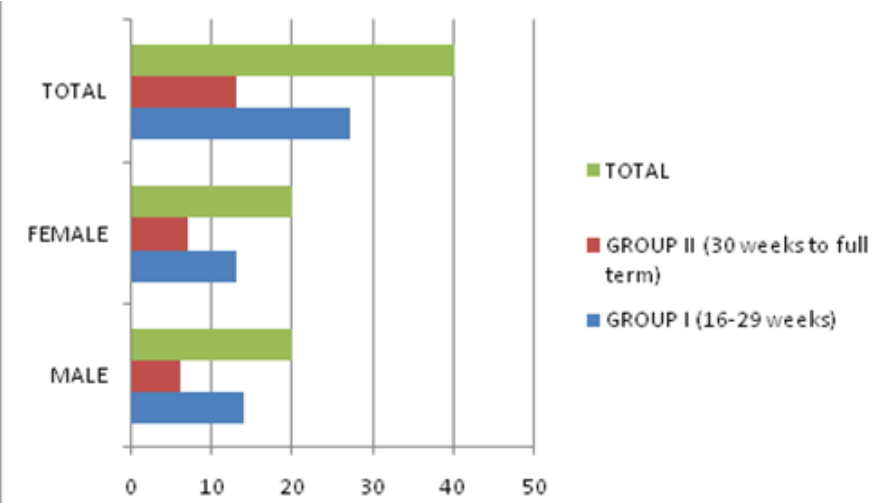

Graph I Sex-wise and gestational age distribution of specimens.

On gross examination the bicuspid orifice was triangular in shape bounded by free margin of leaflets, chordate tendinae and papillary muscles. It presented two cusps. In the present study width and depth of cusps along with frontal and sagittal dimensions of atrioventricular orifice of fetal heart at different gestational ages were recorded in millimeters using paquimeter and the atrioventricular bicuspid valve circumference is calculated by using the formula mentioned in materials and methods. Correlation coefficients between fetal bicuspid valve parameters were analyzed.

The mean and SD values for width and depth of the anterior and posterior cusps were presented in Graph 2 and the values were higher for anterior cusp than for posterior cusp. Mean, SD and P values for different dimensions of bicuspid valve cusps and its circumference in both groups and sexes were presented in Table 1. There is increase in width of anterior cusp, AV bicuspid valve circumference and sagittal dimension in male fetuses and this increase was statistically significant. Width of posterior cusp has increased in both male and female fetuses and this increase was statistically significant.

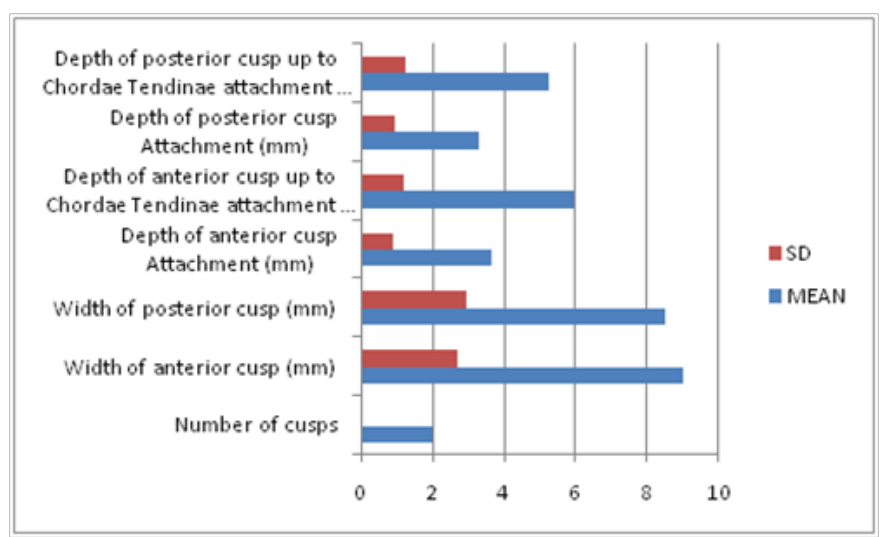

Graph 2 Mean and SD values of width and depth of anterior and posterior cusps of bicuspid valve ( 16 wks.-Full term).

Table I Morphometry of different dimensions of width, frontal, sagittal and atrioventricular circumference of bicuspid valve in $\mathrm{mm}$

\begin{tabular}{|c|c|c|c|c|c|c|}
\hline \multirow{3}{*}{ Measurements } & \multirow{3}{*}{ Sex } & \multicolumn{4}{|c|}{ Age group } & \multirow{3}{*}{ P-values } \\
\hline & & \multicolumn{2}{|c|}{ I (16-29) } & \multicolumn{2}{|c|}{ II (30 weeks- FT) } & \\
\hline & & Mean & SD & Mean & SD & \\
\hline \multirow[t]{2}{*}{ Width of anterior cusp (mm) } & $M$ & 7.55 & 2.41 & 11.24 & 2.38 & $0.01 *$ \\
\hline & $\mathrm{F}$ & 8.6 & 2.51 & 10.83 & 1.99 & 0.06 \\
\hline \multirow[t]{2}{*}{ Width of posterior cusp(mm) } & $M$ & 6.84 & 2.33 & 9.75 & 2.64 & $0.02 *$ \\
\hline & $\mathrm{F}$ & 8.33 & 2.91 & 11.18 & 2.52 & $0.04 *$ \\
\hline \multirow[t]{2}{*}{ Sagittal } & $M$ & 4.91 & 1.47 & 7.21 & 3.16 & $0.04 *$ \\
\hline & $\mathrm{F}$ & 5.44 & 1.51 & 5.95 & 1.66 & 0.05 \\
\hline \multirow[t]{2}{*}{ Frontal } & M & 5.5 & 2 & 7.86 & 3.47 & 0.07 \\
\hline & $\mathrm{F}$ & 5.96 & 1.74 & 5.92 & 1.7 & 0.96 \\
\hline \multirow[t]{2}{*}{ AV bicuspid valve circumference(dimensions) } & M & $|4.6|$ & 8.8 & 32.55 & 23.87 & $0.02 *$ \\
\hline & $\mathrm{F}$ & 17.07 & 8.88 & 18.18 & 8.49 & 0.79 \\
\hline
\end{tabular}

Table 2 shows mean SD and P values of the depths of anterior and posterior cusps in both groups and sexes. This table reveals that depth of anterior cusp attachment, depth of anterior cusp up to Chordae tendinae attachment, posterior cusp attachment and posterior cusp up to Chordae tendinae attachment were significantly increased in male foetuses and this increase was statistically significant. But it is not statistically significant in female fetuses.

(Table 3) (Table 4) reveals that the correlation coefficient of fetal bicuspid valve parameters in Group I and II based on Kruskal-Wallis test. Some of the parameters are positively correlated with one another in both the groups. 
Table 2 Morphometry of different dimension of depth of bicuspid valve in $\mathrm{mm}$

\begin{tabular}{|c|c|c|c|c|c|c|}
\hline \multirow{3}{*}{ Measurements } & \multirow{3}{*}{ Sex } & \multicolumn{4}{|c|}{ Age group } & \multirow{3}{*}{ P-values } \\
\hline & & \multicolumn{2}{|c|}{$I(16-29)$} & \multicolumn{2}{|c|}{ II(30-FT) } & \\
\hline & & Mean & SD & Mean & SD & \\
\hline \multirow{2}{*}{ Depth of anterior cusp attachment } & M & 3.22 & 0.93 & 4.43 & 0.85 & $0.01 *$ \\
\hline & $\mathrm{F}$ & 3.72 & 0.86 & 3.81 & 0.65 & 0.81 \\
\hline \multirow{2}{*}{ Depth of anterior cusp up to Chordae Tendinae attachment } & M & 5.23 & 0.94 & 6.74 & 0.82 & $0.00 *$ \\
\hline & $\mathrm{F}$ & 6.15 & 1.21 & 6.39 & 1.31 & 0.68 \\
\hline \multirow{2}{*}{ Depth of posterior cusp Attachment } & M & 2.89 & 0.88 & 4.15 & 1.23 & $0.02 *$ \\
\hline & $\mathrm{F}$ & 3.16 & 0.86 & 3.52 & 0.52 & 0.32 \\
\hline \multirow{2}{*}{ Depth of posterior cusp up to Chordae Tendinae attachment } & M & 4.59 & 1.04 & 6.32 & 1.26 & $0.00 *$ \\
\hline & $\mathrm{F}$ & 5.32 & 1.21 & 5.54 & 1.05 & 0.69 \\
\hline
\end{tabular}

Table 3 Correlation coefficient of fetal bicuspid valve parameters in (16-29)weeks of gestational age

\begin{tabular}{|c|c|c|c|c|c|c|c|c|c|c|c|}
\hline Age & & & AV_Total & DAA & DACT & DPA & DPCT & Frontal & Sagittal & WP & WA \\
\hline \multirow[t]{2}{*}{ Age } & $*_{r}$ & & 0.391 & 0.268 & 0.4 & 0.36 & $0.4 I$ & 0 & 0.413 & $0.67 I * *$ & $0.691 * *$ \\
\hline & $P$ & & 0.0438 & 0.177 & 0.04 & 0.07 & 0.04 & 0 & 0.032 & I.00E-04 & 0 \\
\hline $\mathrm{AV}_{-}$ & $*_{r}$ & 0.391 & & $0.38 I$ & $0.517^{*}$ & 0.39 & 0.46 & $0.952^{* *}$ & $0.908 * *$ & 0.376 & 0 \\
\hline \multirow[t]{2}{*}{ Total } & $P$ & 0.044 & & 0.05 & 0.01 & 0.04 & 0.01 & $<0.0001$ & $<0.0001$ & 0.053 & 0 \\
\hline & $*_{r}$ & 0.268 & 0.381 & & $0.634^{*}$ & $0.792^{* *}$ & 0.61 & 0 & 0.353 & 0.391 & 0 \\
\hline \multicolumn{12}{|l|}{ DAA } \\
\hline & $P$ & 0.177 & 0.0502 & & 0 & $<0.0001$ & 0 & 0 & $0.07 I$ & 0.044 & 0 \\
\hline & $*_{r}$ & 0.399 & $0.517 *$ & $0.634 *$ & & $0.714^{* *}$ & $0.856 * *$ & 0 & 0.459 & 0.534 & 1 \\
\hline \multicolumn{12}{|l|}{ DACT } \\
\hline & $P$ & 0.039 & 0.0057 & $4.00 \mathrm{E}-04$ & & $<0.0001$ & $<0.0001$ & 0 & 0.016 & 0.004 & 0 \\
\hline & $*_{r}$ & 0.358 & 0.393 & $0.792 * *$ & $0.714^{* *}$ & & $0.822^{* *}$ & 0 & 0.363 & $0.597^{*}$ & $0.516^{*}$ \\
\hline \multicolumn{12}{|l|}{ DPA } \\
\hline & $P$ & 0.067 & 0.0426 & $<0.0001$ & $<0.0001$ & & $<0.0001$ & 0 & 0.063 & 0.001 & 0 \\
\hline & $*_{r}$ & 0.406 & 0.464 & 0.606 & $0.856^{* *}$ & $0.822^{* *}$ & & 0 & 0.393 & $0.64 I^{*}$ & I \\
\hline \multicolumn{12}{|l|}{ DPCT } \\
\hline & $P$ & 0.036 & 0.0148 & $8.00 \mathrm{E}-04$ & $<0.0001$ & $<0.0001$ & & 0 & 0.042 & $3.00 \mathrm{E}-04$ & 0 \\
\hline & $*_{r}$ & 0.359 & $0.952 * *$ & 0.345 & 0.49 & 0.36 & 0.44 & & $0.770 * *$ & 0.332 & 0 \\
\hline \multicolumn{12}{|l|}{ Frontal } \\
\hline & $\mathrm{P}$ & 0.066 & $<0.0001$ & 0.078 & 0.01 & 0.06 & 0.02 & & $<0.0001$ & 0.091 & 0 \\
\hline & $*_{r}$ & 0.413 & $0.908^{* *}$ & 0.353 & 0.46 & 0.36 & 0.39 & $0.770 * *$ & & 0.423 & 0 \\
\hline \multicolumn{12}{|l|}{ Sagittal } \\
\hline & $\mathrm{P}$ & 0.032 & $<0.0001$ & 0.071 & 0.02 & 0.06 & 0.04 & $<0.0001$ & & 0.09 & 0 \\
\hline & $*_{r}$ & 0.67 I** & 0.376 & 0.391 & 0.53 & $0.597 *$ & $0.64 I *$ & 0 & 0.423 & & $0.920 * *$ \\
\hline \multicolumn{12}{|l|}{ WP } \\
\hline & $\mathrm{P}$ & I.00E-04 & 0.0533 & 0.044 & 0 & 0 & 0 & 0 & 0.09 & & $<0.0001$ \\
\hline & $*_{r}$ & $\left.0.69\right|^{* *}$ & 0.382 & 0.326 & 0.59 & $0.516 *$ & $0.586 *$ & 0 & 0.395 & $0.920 * *$ & \\
\hline \multicolumn{12}{|l|}{ WA } \\
\hline & $P$ & I.00E-04 & 0.049 & 0.097 & 0 & 0.01 & 0 & 0 & 0.042 & $<0.0001$ & \\
\hline
\end{tabular}

$*_{r}$, correlation coefficient; $P$, $p$ value; DAA, depth of anterior cusp attachment; DPA, depth of posterior cusp attachment;WP, width of posterior cusp;WA, width of anterior cusp; DPA, depth of posterior cusp up to code tendon attachmenT;AV total, atrioventricular circumference 
Table 4 Correlation Coefficient of Fetal Bicuspid Valve Parameters in Group-II

\begin{tabular}{|c|c|c|c|c|c|c|c|c|c|c|c|}
\hline Age & & & AV_Total & DAA & DACT & DPA & DPCT & Frontal & Sagittal & WP & WA \\
\hline \multirow{2}{*}{ Age } & $*_{r}$ & & 0.583 & 0.6 & 0.21 & 0.69 & I & 0.374 & 0.597 & 0 & 0.419 \\
\hline & $P$ & & 0.036 & 0.03 & 0.48 & 0.01 & 0 & 0.208 & 0.031 & 0 & 0.154 \\
\hline \multirow{2}{*}{ AV_TOTAL } & $*_{r}$ & 0.583 & & 0.66 & 0.44 & 0.79 & $0.654^{*}$ & $0.948 * *$ & $0.939 * *$ & 0 & 0.608 \\
\hline & $P$ & 0.0364 & & 0.01 & 0.14 & 0 & 0 & $<0.0001$ & $<0.0001$ & 0 & 0.0275 \\
\hline \multirow{2}{*}{ DAA } & $*_{r}$ & 0.601 & 0.661 & & 0.66 & $0.930 * *$ & $0.843^{*}$ & 0.514 & 0.619 & 0 & 0.56 \\
\hline & $P$ & 0.0298 & 0.014 & & 0.01 & $<0.0001$ & 0 & 0.073 & 0.024 & 0 & 0.0464 \\
\hline \multirow{2}{*}{ DACT } & $*_{r}$ & 0.214 & 0.436 & 0.66 & & 0.64 & $0.857^{*}$ & 0.423 & 0.333 & I & 0.552 \\
\hline & $P$ & 0.4821 & 0.137 & 0.01 & & 0.02 & 0 & 0.15 & 0.267 & 0 & 0.0505 \\
\hline \multirow{2}{*}{ DPA } & $*_{r}$ & 0.687 & 0.791 & $0.930^{* *}$ & 0.64 & & $0.88 I^{* *}$ & $0.64 I$ & $0.720 *$ & I & 0.58 \\
\hline & $P$ & 0.0095 & 0.001 & $<0.0001$ & 0.02 & & 0 & 0.018 & 0.006 & 0 & 0.0377 \\
\hline \multirow{2}{*}{ DPCT } & $*_{r}$ & 0.549 & 0.654 & $0.843^{*}$ & $0.857^{*}$ & $0.88 I^{* *}$ & & 0.585 & 0.515 & I & 0.532 \\
\hline & $P$ & 0.0522 & 0.015 & 0 & 0 & 0 & & 0.036 & 0.072 & 0 & 0.0613 \\
\hline \multirow{2}{*}{ Frontal } & $*_{r}$ & 0.374 & $0.948 * *$ & 0.51 & 0.42 & 0.64 & I & & $0.835^{*}$ & 0 & 0.544 \\
\hline & $P$ & 0.2076 & $<0.0001$ & 0.07 & 0.15 & 0.02 & 0 & & 4.00E-04 & 0 & 0.0544 \\
\hline \multirow{2}{*}{ Sagittal } & $*_{r}$ & 0.597 & $0.939 * *$ & 0.62 & 0.33 & $0.720 *$ & I & $0.835 *$ & & 0 & 0.627 \\
\hline & $P$ & 0.0312 & $<0.0001$ & 0.02 & 0.27 & 0.01 & 0 & $4.00 \mathrm{E}-04$ & & 0 & 0.0218 \\
\hline \multirow{2}{*}{ WP } & $*_{r}$ & 0.21 & 0.476 & 0.4 & 0.64 & 0.53 & I & 0.434 & 0.487 & & 0.675 \\
\hline & $P$ & 0.4918 & 0.1 & 0.18 & 0.02 & 0.07 & 0 & 0.138 & 0.092 & & 0.0114 \\
\hline \multirow{2}{*}{ WA } & $*_{r}$ & 0.419 & 0.608 & 0.56 & 0.55 & 0.58 & 1 & 0.544 & 0.627 & I & \\
\hline & $P$ & 0.154 & 0.028 & 0.05 & 0.05 & 0.04 & 0 & 0.054 & 0.022 & 0 & \\
\hline
\end{tabular}

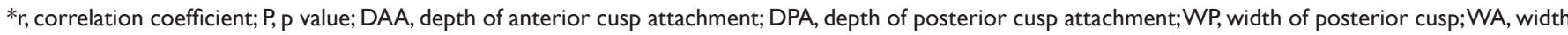
of anterior cusp; DPA, depth of posterior cusp up to code tendon attachmenT;AV total, atrioventricular circumference

\section{Discussion}

In the literature, there are no reports on bicuspid valve morphology and morphometry in developing human fetuses. Those that were reported were in adults. Hence the present study could not be compared. The frontal and sagittal dimensions of the bicuspid valve increased in both sexes with an increase in gestational age in the present study, which corresponds to data of Skwarek et al. ${ }^{5}$ in adults. Those studies that reported on the shape of atrioventricular orifice in literature in adults were based on the application of special formula by Skwarek et al., ${ }^{4}$ which was followed by Gunnal et al. ${ }^{6}$ on left atrioventricular orifice as was done in the present study. A recent study in Northwest part of Iranian adult population by Shabnam Mohammadi ${ }^{7}$ reported significantly higher circumference and area of mitral valve in males than in females as observed in our study on fetuses.

The present study is the first to report on the dimensions of the mitral valve length and depth of the anterior and posterior leaflet in human fetal cadavers. The present study indicates that the bicuspid annular diameter and dimension of the valve orifice closely correlated with age, depth of anterior, posterior and width of anterior and posterior cusps in both groups.

\section{Conclusion}

Present study indicated that bicuspid annular diameter and the dimensions of annular orifice positively correlate with one another and with age of the fetus in both sexes. Bicuspid morphology gained importance with increase in the incidence of cardiac surgeries. It may provide new information about normal and abnormal function of the mitral valve apparatus. The normal value of the mitral valve may serve an important role in valve replacement surgeries. As there were no reported studies on fetal bicuspid valve parameters the present study will form a baseline data on gestational age related normal bicuspid valve complex in Indian fetal population.

\section{Acknowledgements}

None.

\section{Conflict of interest}

Author declares that there is no conflict of interest.

\section{References}

1. Du Plessis LA, Merchant P. The anatomy of the mitral valve and its associated structures. Thorax. 1964;19:221-227. 
2. Gopal MR, Taha A, John V, et al. Pediatric valve replacement, 15 years' experience in Oman. Ind $J$ Thorac Cardiovasc Surg. 2006;22(3):173-177.

3. Ludwig J. Handbook of autopsy practice. 3rd ed. Cardiovascular system. New Jersey: Human Press Inc; 2002:45-52.

4. Skwarek M, Grzybiak M, Kosinski A, et al. Notes on the morphology of the tricuspid valve in the adult human heart. Folia Morphol (Warsz). 2004;63(3):319-324.
5. Skwarek M, Hreczecha J, Dudziak M, et al. Morphometric features of the right atrioventricular orifice in adult human hearts. Folia Morphol (Warsz). 2008;67(1):53-57.

6. Gunnal SA, Farooqui MS, Wabale RN. Study of mitral valve in human cadaveric hearts. Heart Views. 2012;13(4):132-135.

7. Mohammadi S, Hedjazi A, Sajjadian M, et al. Study of the normal heart size in Northwest part of Iranian population: a cadaveric study. $J$ Cardiovasc Thorac Res. 2016;8(3):119-125. 